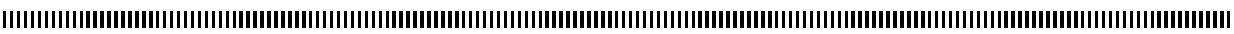

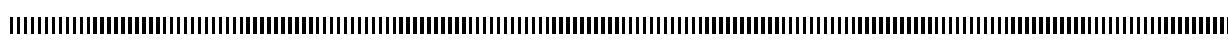

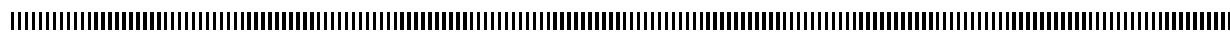

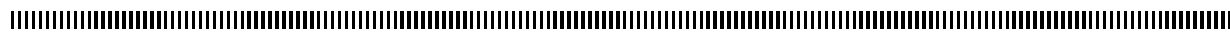

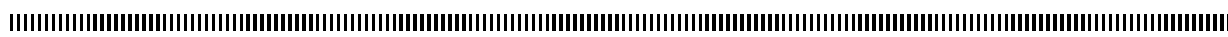

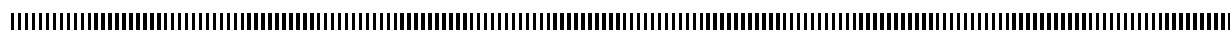

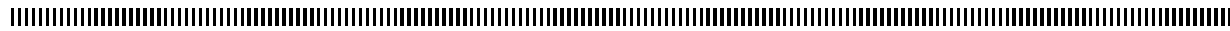
||||||||||||||||||||||||||||||||||||||||||||||||||||||||||||||||||||||||||||||||||||||||||||||||||||||||||||||||||||||||||||||||||||||||||||||||||||||||||||||||||||||||||||||||||||||||||||||||||||||||||||||||

\title{
Modélisation des interactions de coopération dans la conduite d'un projet de simulation
}

\author{
Ahmed Korichi*, Brahim Belattar** \\ * Département de Mathématique et d’Informatique, Université de Ouargla 30000 Algérie. \\ ahmed.korichi@gmail.com \\ ** Département d’Informatique, Université de Batna 05000 Algérie. \\ belattarb@yahoo.com
}

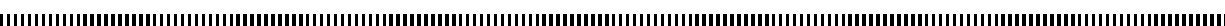

RÉSUMÉ. Cette étude a été menée dans le cadre d'un projet de recherche intitulé «TCAO et Simulation : Vers une plate-forme d'analyse et de conception de systèmes de production orientée groupe». Ce projet a pour but d'analyser les pratiques de coopération lors de la conduite d'un projet de modélisation et de simulation d'un système de production, puis de spécifier et développer un collecticiel dans le but d'ajouter la dimension de groupe aux outils de simulation. Dans cet article, nous définissons et nous présentons une modélisation des interactions de coopération dans la conduite d'un un projet de simulation à l'aide de Denver Model. Nous illustrons notre approche en présentant notre expérience avec le système BSCW pour valider notre modélisation.

ABSTRACT. This study was carried out within the framework of a research project entitled «CSCW and Simulation: Toward a group-oriented platform of analysis and design of production systems». The object of this project is to analyze the cooperation practices during the conduct of a project of modeling and simulation of a production system, then to specify and develop a simulation groupware with an aim of adding the group dimension to simulation tools. In this paper, we define and we present a modeling of cooperation interactions in the conduct of cooperative simulation project using the Denver Model. We illustrate our approach by presenting our experiment with BSCW system to validate our modeling.

MOTS-CLÉS : Simulation, Modélisation, Simulation coopérative, BSCW, TCAO, Denver Model.

KEYWORDS: Simulation modeling, cooperative simulation, BSCW, CSCW, Denver Model.

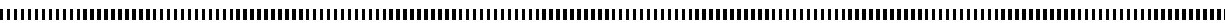

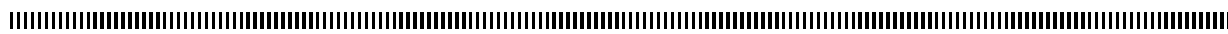

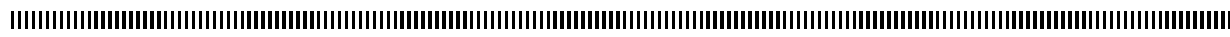

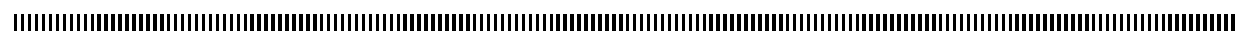

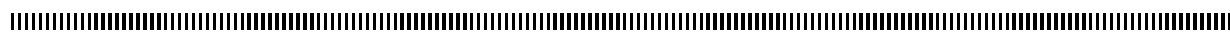

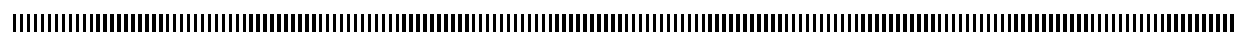

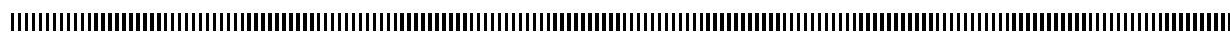

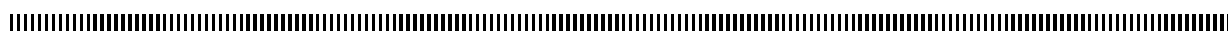

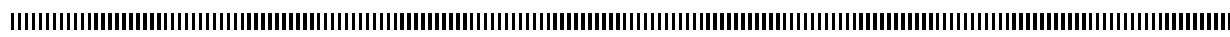
|

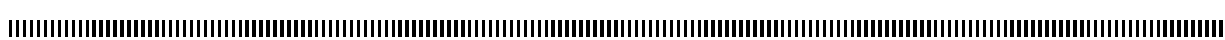




\section{Introduction.}

L'informatisation de la société est inéluctable, qu'on le veuille ou non. Le réseau informatique Internet conquiert chaque jour une place plus importante chez les professionnels qui profitent de son faible coût d'exploitation, de sa facilité d'usage et de son universalité. Cette révolution emboîte le pas à une révolution plus ancienne, celle de l'informatisation des années 1980, et c'est tout logiquement que se trouvent renforcés les échanges et questionnés les protocoles de collaboration et les pratiques coopératives.

Les logiciels de première génération, logiciels dits « métier » ne couvrant que les besoins d'une pratique professionnelle, n'ont pas amené de modifications profondes dans l'organisation des pratiques professionnelles [10]. Cependant, la démocratisation de l'accès à Internet, le nombre croissant de réseaux d'entreprises et de particuliers (i.e. Intranet et Internet) font que les utilisateurs s'orientent de plus en plus vers un mode de travail communautaire et géographiquement ouvert. Ces derniers deviennent de plus en plus exigeants, et attendent de l'outil informatique qu'il les assiste dans leur travail quotidien. La solution qui a consisté dans le passé à investir dans le développement d'applications typiquement centralisées et mono-utilisateur est en passe d'être écartée. La prise en compte de la dimension de groupe dans la conception des applications est devenue un facteur prédominant [02]. Certaines applications qui par le passé étaient proposées sous forme d'applications mono-utilisateur (Editeur de texte, Outil de C.A.O.) existent aujourd'hui sous forme d'application à l'usage d'un groupe (Groupware Application).

Dans ce contexte l'objectif de nos travaux est l'adaptation des résultats des recherches issus du domaine du travail coopératif assisté par ordinateur (TCAO) par vocation généraliste, à un domaine spécifique, la conduite d'un projet de simulation. Nous sommes motivés pour définir et modéliser les interactions de coopération dans la conduite d'un projet de simulation dans le but de contribuer à la conception d'une nouvelle génération d'outils de simulation basés sur le travail de groupe.

Dans la première partie de cet article, nous présentons la méthode de conduite d'un projet de simulation et nous justifions l'adéquation de cette méthode avec les concepts du TCAO. A partir de cette méthode nous définissons les modes de coopération possible dans un projet de simulation. Dans la seconde partie, nous caractérisons ces modes de coopération à l'aide de la méthode de Denver Model. Enfin, dans la dernière partie, nous présentons notre expérience avec le Système BSCW pour instancier et valider notre modélisation.

\section{A RIM A}




\section{Simulation et Travail Coopératif.}

\subsection{Dimension de groupe dans un projet de simulation}

D’un point de vue externe, on a souvent tendance à voir en l'ingénieur ou l'analyste le seul acteur d'un projet de simulation surtout si celui-ci maîtrise bien le logiciel de simulation qui lui sert de support pour la codification et l'évaluation du modèle. Or, sur le plan pratique, beaucoup de personnes seront sollicitées pour alimenter le projet en données ou en expertises afin qu'il aboutisse (statisticien, chef d'atelier, ingénieur de méthodes, programmeur, client de l'étude, etc.). Il est aussi communément reconnu que pour pouvoir utiliser correctement et intelligemment les méthodes de simulation, il faut disposer de connaissances plus ou moins solides dans des domaines variés (Probabilités et Statistiques, Modélisation, Programmation, etc.). Malheureusement, il est souvent rare pour ne pas dire impossible de trouver au sein de l'entreprise un cadre possédant à lui seul toutes les connaissances requises pour pouvoir mener à termes un projet de simulation sans avoir recours aux connaissances d'un certain nombre d'individus internes ou externes à son entreprise. Tout projet de simulation quelle que soit son envergure fait intervenir donc de près ou de loin de nombreuses personnes avec des compétences et des rôles différents [01]. Par conséquent, un projet de simulation quelle que soit son envergure, ne peut et ne pourra jamais être considéré comme l'œuvre d'une personne isolée et ce même si les attributions de rôle dans le projet ne sont pas faites de manière explicite.

\subsection{Conduite d'un projet de simulation coopératif}

Théoriquement un travail en groupe peut s'organiser selon les deux modes extrêmes de travail que sont la sous-traitance et la co-traitance [13]. Le mode de travail effectif est souvent une combinaison de ces deux modes. Dans les deux cas, le chef de projet décompose le travail à réaliser en parties et le décrit sous forme d’objectifs et de contraintes. Il fournit le sous-ensemble de départ et le contexte à chaque participant ou équipe. Chaque participant travaille sur son sous-ensemble en respectant les objectifs et les contraintes imposés puis fournit au chef de projet le résultat du travail. Le chef de projet réceptionne les sous-ensembles réalisés, les contrôles et effectue la fusion. Dans le mode de sous-traitance, toutes les contraintes doivent être parfaitement définies afin que chaque participant puisse travailler de façon autonome. La coopération se limite aux interactions ponctuelles et aux phases de validation et de transmission des informations. Dans le mode de co-traitance, les interactions entre participants ont lieu durant tout le processus. Celles-ci permettent de détecter et de résoudre au fur et à mesure les problèmes. D'une façon théorique, lors d'un travail à plusieurs personnes, les 
phases d'analyse, de conception et de validation peuvent être menées soit de façon séquentielle (pure ou itérative), soit de façon concourante. Bien sûr, la co-traitance ne peut pas s'effectuer en mode séquentiel pur: il n'y aurait pas d'interactions possibles entre les participants.

Cette organisation générale du travail en groupe est parfaitement applicable dans le domaine de la simulation. En effet, un projet de ce domaine consiste en un ensemble de tâches chacune d'elles nécessitant des compétences particulières et dont certaines ne peuvent avoir lieu que si d'autres ont été achevées [06]. Ces tâches sont accomplies par un collectif d'acteurs placé sous la responsabilité d'un chef de projet et qui coopèrent à l'occasion de ce projet visant un résultat défini, connu et mesurable. Ce collectif d'acteurs, dans sa taille et dans sa configuration, varie d'un projet à un autre. Cette variation n'est pas seulement liée à la taille du projet et à son coût, bien qu'elle dépende largement de ces deux facteurs, mais également à sa complexité fonctionnelle et technique; ainsi, la simulation d'un avion fait intervenir des dizaines de spécialistes et des personnes compétentes. À l'opposé, la simulation d'un moteur électrique ne requiert dans la majorité des cas que peu d'acteurs : un informaticien et un électrotechnicien. Les principaux participants dans l'équipe chargée l'ensemble de la réalisation du projet, sont les informaticiens (Ingénieur spécialisé en simulation, Analyste, Programmeur, Agents de saisie... etc.), le personnel de métiers (Ingénieur de méthodes, Techniciens, Agents de métier), les mathématiciens (Statistiques et Probabilités) et les experts consultants (experts en simulation, experts en métiers) [17].

Un projet de simulation peut être décomposé en phases et en études de sousensembles dont la complexité est plus facilement maîtrisable possédant entre eux des relations et des contraintes. Les sous-ensembles correspondent à des techniques éventuellement différentes (modélisation, statistique et probabilité, programmation, de métiers) [04]. Ce découpage en phases et en sous-ensembles maîtrisables est essentiel à sa conduite, de procéder à sa planification et donc à son bon aboutissement et à sa réussite. Elle permette aussi de bâtir les différents scénarios de coopération possible entre les acteurs du projet.

La mise en œuvre d'un projet de simulation (informatique) se décline en plusieurs étapes à suivre avec le plus grand soin. Elle débute par des actions touchant à la formalisation des objectifs et du cahier des charges. Vient ensuite la planification (délais, finance, etc.), ainsi que l'acquisition du matériel et logiciel informatique, le recrutement de personnes compétentes et l'attribution des rôles et objectifs dévolus à chacune. Lors des déploiements en tant que tels, la démarche se poursuit par la coordination et le suivi des équipes et des travaux, qui peut d'ailleurs s'effectuer par le biais de mécanismes de supervision et de travail collaboratif [08].

D'autres parts, la conduite d'une étude de simulation proprement dites comprend trois étapes principales (Figure 01) : l'analyse du problème, la construction du modèle et l'exploitation de ce modèle.

\section{A RIM A}




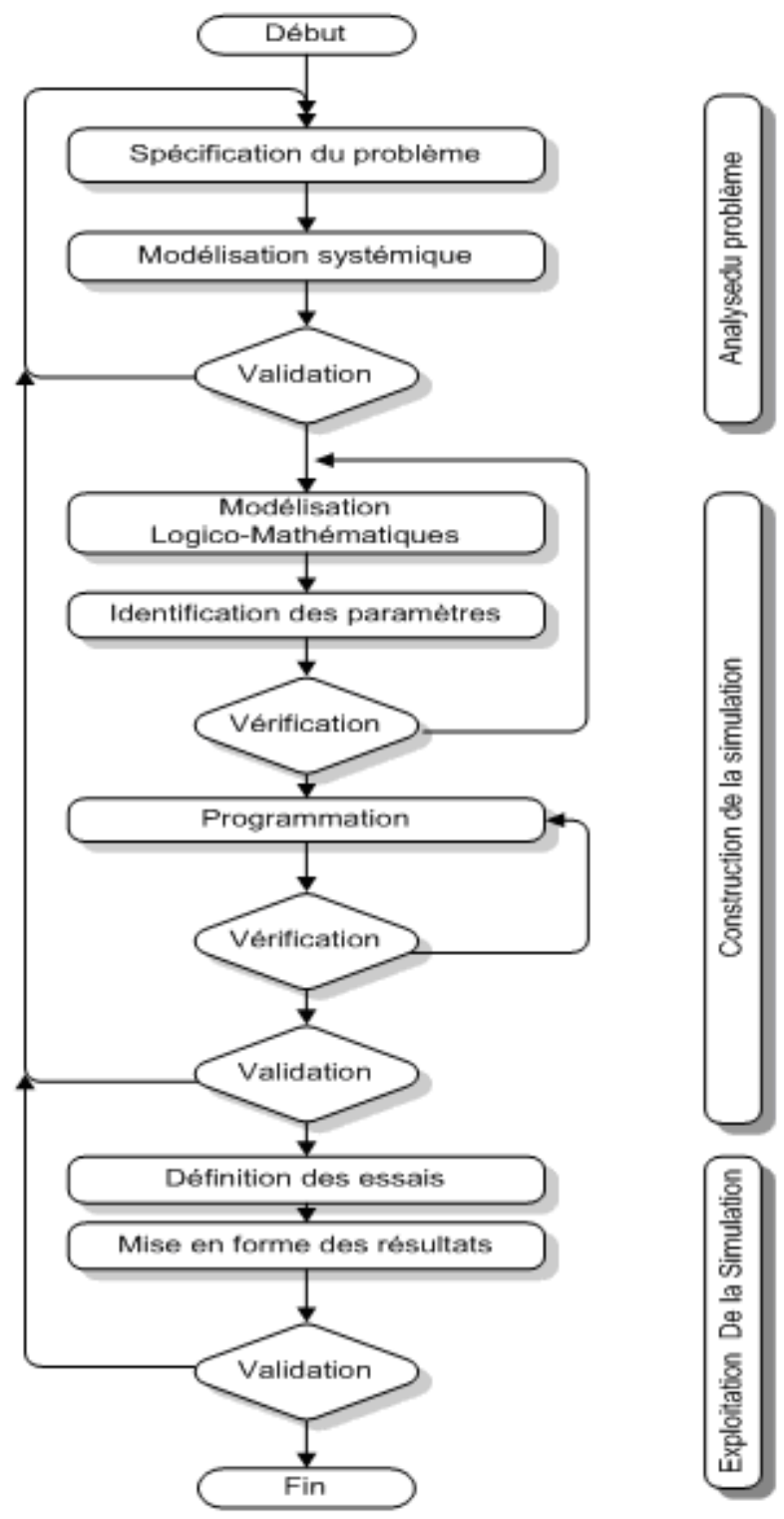

Figure 1 : Conduite d'une étude de simulation [07] 
La première étape permet de préciser le contexte de l'étude. Elle comprend l'identification du problème; la spécification des objectifs, la réalisation d'une première ébauche du modèle qui a pour but d'en délimiter les frontières et de spécifier les données dont on a besoin et la validation de ce modèle auprès de l'utilisateur. Le but à atteindre dans cette étape est de construire un modèle valide tout en restant cohérent avec les objectifs de l'étude. L'étape de construction d'un modèle comprend la modélisation logico-mathématique qui peut être facilitée par un outil graphique, et la programmation proprement dite. Il est important dans cette étape de construire un programme facilement modifiable et en particulier de distinguer clairement le système réel, le système de conduite et le système d'information. Dans sa première itération, cette étape se termine par une validation qui consiste à comparer le comportement du modèle avec celui du système réel qu'il est censé représenter. Dans la troisième étape, le modèle validé peut servir à l'évaluation du comportement dynamique du système. Cette étape nécessite une définition précise de la campagne d'exploitation (quelles hypothèses veut-on vérifier, dans quel contexte), la production de mesures par la simulation proprement dite, la mise en forme et la comparaison des résultats obtenus aux objectifs poursuivis. S'ils n'ont pas été atteints, de nouveaux scénarios sont proposés et testés jusqu'à satisfaction. Dans la mesure où la plupart des modèles comportent des aléas, l'étape d'exploitation nécessite que soient déterminés avec rigueur la durée de la simulation et le nombre de réplications (exécutions du modèle de simulation); elle fait appel aux outils statistiques afin de caractériser le comportement du modèle : calcul d'intervalles de confiance, de coefficients de corrélation,

Grâce à cette présentation de la conduite d'un projet de simulation, nous a permis d'établir une modélisation des interactions de coopération des participants d'un projet de simulation. A partir de cette modélisation, il est alors possible de spécifier plus finement les tâches coopération dans projet de simulation et ainsi d'améliorer les outils de simulation existants pour que l'utilisateur soit plus performant dans son projet.

\subsection{Modes de coopération}

Pour construire une application de groupe en générale, il est nécessaire d’identifier les modes de coopération selon lesquels les membres pourraient interagir avec l'application et communiquer ainsi entre eux. Globalement, une application de groupe, doit supporter plusieurs canaux de communication qui permettront d'assurer respectivement [02]:

Une communication Homme Machine avec les applications classiques utilisées localement par chaque membre (traitement de texte, Tableur, etc.). Parmi ces applications figure nécessairement l'outil de modélisation et de simulation. 
Une communication Homme Machine qui met en jeu la coopération et dont le but essentiel est la manipulation coordonnée des objets partagés par les membres du groupe et ce dans un vrai mode multi-utilisateurs.

Une communication Homme Homme qui offre aux membres du groupe plusieurs modes de conversation : messagerie, chat, stick up, vidéophone.

Dans sa thèse de doctorat, Tarpin-Bernard [13] a identifié quatre modes de coopération possible pour un travail de groupe. Ces modes de coopération sont parfaitement applicables dans la conduite d'un projet de simulation :

o La coopération asynchrone : Dans le contexte d'une organisation, la coopération asynchrone correspond au mode de travail autonome. Chacun travaille de son côté puis soumet des rapports, comptes-rendus, etc. aux interlocuteurs adéquats qui, plus tard, feront part de leurs analyses. Dans ce mode de coopération la rencontre est informelle, et chacun travaille indépendamment les uns des autres, mais il n'est pas exclu qu'un participant puisse utiliser un travail fait par un autre (modèle, code, etc.) Les différents participants interagissent dans le projet en échangeant les données et en travaillant quand ils le peuvent (à des instants généralement différents : sans co-temporalité).

o La coopération en session : La coopération en session correspond à l'acte de présence au sein de l'organisation et peut être rapprochée de la notion d'astreinte. En effet, dans un tel mode, les participants connaissent la liste de leurs interlocuteurs potentiels à un instant donné. Dans le cas de l'astreinte, cette liste est prédéfinie par le chef de projet. Elle se met à jour à partir des agendas individuels. A titre d'exemple, le téléphone peut représenter un outil type de la coopération en session. En effet, la communication téléphonique ne peut s'établir si les interlocuteurs ne sont pas présents simultanément. Cependant, l'informatique permet d'envisager des outils beaucoup plus puissants que le téléphone et qui permettent d'abolir d'une façon plus nette les barrières spatiales séparant les différents participants d'un projet. Dans ce mode de coopération les différents participants travaillent en même temps, mais de façon autonome. Ils sont accessibles pour communiquer (en co-temporalité), mais sans partager de façon visuelle les objets de leurs discussions.

o La coopération en réunion : La notion de réunion introduite dans la coopération en réunion correspond exactement à celle qui existe dans le cadre d'un projet. De fait, elle est planifiée et elle définit des rôles spécifiques à chaque participant. De plus, elle porte sur un ensemble d’objets clairement identifié (Modèle, Programme, 
Cadre d'expérimentation, document, etc.) qui doivent être partagés par l'ensemble des membres de la réunion. Contrairement à la coopération asynchrone, la coopération en réunion impose la participation active (ex: réponse immédiate aux questions). Dans ce mode coopération des participants clairement identifiés travaillent et communiquent en co-temporalité tout en partageant les objets de leurs travaux et discussions. Ils se voient attribuer des rôles en relation avec le but de la réunion. L'organisation de leurs interventions est régie par un mécanisme de type "tour de parole"

o La coopération étroite : La coopération étroite correspond à une vision plus précise de la coopération. Elle permettre une interaction maximale entre les participants dans un monde cohérent simulant la réalité. Cette forme de coopération se rapproche de la notion de travail coopératif à la planche. C'est notamment dans ce cadre que l'on peut imaginer de nouvelles formes de coopération non encore exploitées. La puissance de cette coopération réside dans la liberté d'action qu'engendre la possibilité d'agir finement et simultanément sur des objets d'un monde virtuel global. Dans ce mode coopération les participants peuvent travailler, communiquer et interagir en temps réel sur tous les objets partagés du projet. Les conséquences de leurs interventions sont directement gérées au niveau des objets manipulés.

Nous appliquons dans la suite le modèle Denver pour caractériser ces modes de coopération, du point de vue des interactions, situations et protocoles.

\section{Modèles des interactions}

\subsection{Modèle de Denver}

Le modèle de Denver offre un cadre d'évaluation et de conception des fonctionnalités pour les collecticiels. Il résulte de la mise en commun de travaux de quatorze participants à l'atelier sur « la conception et l'évaluation des collecticiels », tenu pendant la conférence ACM CHI (Computer Human-Interaction) en 1995 à Denver (Colorado) [12]. Ce modèle comporte trois sous-modèles qui forment trois couches superposées : en haut, un modèle des objectifs et des besoins (System Requirements), au milieu un modèle de conception (Design Model) et en dessous un modèle technologique (Technology). Le développement principal du modèle s'est porté sur le modèle intermédiaire, le modèle de conception. Le sous-modèle de conception définit un cadre de conception d'un collecticiel, par cinq catégories de caractéristiques [09] : 
Les Personnes (People) : cette catégorie inclut les caractéristiques des utilisateurs, des groupes et des rôles qu'une personne peut avoir au sein d'un groupe. Une personne peut être identifiée par son nom, son adresse, son numéro de téléphone, son métier, etc. Son rôle peut être identifié par rapport à un groupe, par rapport à la gestion du collecticiel, ou d'autres facteurs plus subjectifs. Chaque personne peut appartenir à plusieurs groupes et tenir un rôle différent dans chacun d'eux. Les caractéristiques du groupe vont de « stable et homogène » à « instable et hétérogène ». Les auteurs du modèle de Denver notent que la représentation du groupe reste une question ouverte. Dans le cas d'un projet de simulation, les groupes formés sont caractérisés par : Une certaine stabilité, homogénéité, de par le but commun, la taille d'un groupe qui est a priori petite dépendant de l'envergure du projet.

Les Artéfacts (Artifacts) : cette catégorie se réfère à tous les objets manipulés, produits ou consommés pendant l'interaction. Il en existe cinq types génériques : le texte, le son, l'image temporel, l'image statique et l'élément de calcul. Ces types peuvent se combiner à l'intérieur d'un artefact qui est alors composite. Les artefacts possèdent des attributs comme la cotemporalité, la révisabilité, la possibilité d’en faire un compte-rendu, etc. Par exemple les différents modèles conceptuels, l'inventaire des besoins en données sur le système réel, les différents modèles opérationnels, les différents cadres d'expérimentations et la documentation du projet constituent les résultats produits par les équipes du projet.

Les Tâches et Activités (Tasks and Activities) : cette catégorie regroupe les caractéristiques des tâches et activités réalisables par le biais du collecticiel. Le modèle de Denver distingue quatre niveaux : les objectifs, les tâches et scénarios, les activités et les opérations. Les objectifs correspondent aux objectifs les plus globaux. Dans le contexte de simulation, les objectifs sont par exemple «évaluation de performances d'un système ", " respecter les délais et les coûts tout en garantissant la qualité d'étude souhaité ", etc. À un niveau inférieur, les tâches sont par exemple «concevoir les modèles», "valider des documents », etc. Les activités se combinent pour former des tâches : " actualiser sa base de modèles », " programmer des modèles », " échanger des idées » en sont des exemples. Enfin, les opérations sont des manipulations élémentaires sur des artefacts.

Les Situations d'Interaction (Interactive Situations) : Elles définissent la relation des participants entre eux, leur relation au temps et leur relation à l'espace. Les auteurs du modèle les représentent par une étoile à cinq axes qui correspondent respectivement à cinq notions : la dépendance, la temporalité, la coordination temporelle, le localisation du groupe et la taille du groupe. Ainsi, les caractéristiques du collecticiel permettent de 
donner la position de cinq points appartenant à chacun de ces axes (le degré d'entropie maximum est situé au centre de l'étoile). Les cinq points obtenus, reliés entre eux, forment une figure à cinq côtés qui est une des « signatures » du collecticiel.

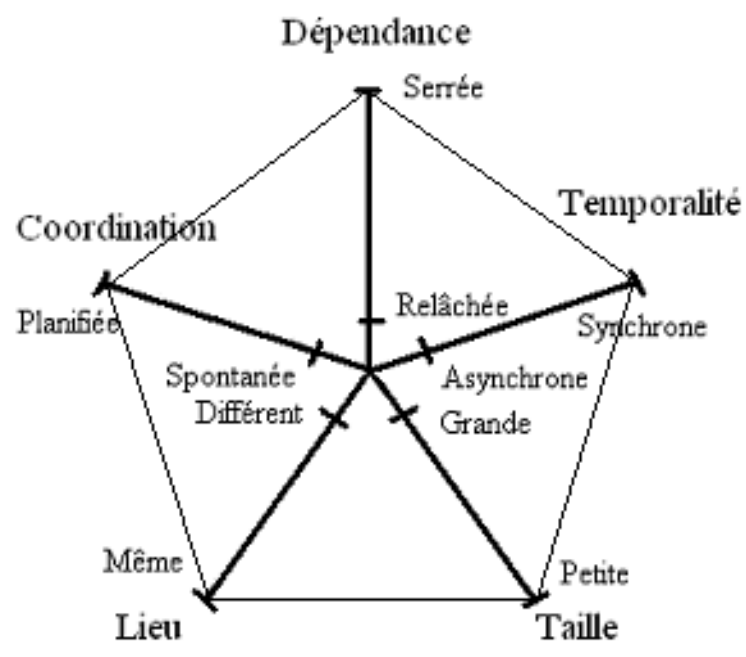

Figure 2 : Les cinq axes caractérisant la situation d'interaction [12].

Les Protocoles Sociaux de l'Interaction (Interactive Social Protocols) : un protocole social se réfère aux séquences possibles d'échange de signaux et d'informations qui déterminent et identifient les conflits et ses résolutions. Les auteurs du modèle utilisent la même représentation en étoile à cinq axes que pour les situations interactives. Ici les axes correspondent au style de réunions (meeting style), à la détection de conflits et à leur résolution (contention detection and resolution), à la taille du groupe, à la réglementation des interventions (floor control) et à la familiarité (formality of address). Le style de réunion décrit la façon dont les réunions entre participants se déroulent (unidirectionnel ou multidirectionnel). La caractéristique intitulée « détection des conflits et résolution » décrit la capacité du collecticiel à découvrir les conflits éventuels au sens large, le conflit est une situation de concurrence ou d'opposition des participants et le moment de leur résolution. La réglementation des interventions se réfère à la façon plus ou moins dirigée d'agir ou par exemple de " prendre la parole ». La familiarité correspond quant à elle à la façon plus ou moins formelle et protocolaire qu'on les participants de s’adresser les uns aux autres. 


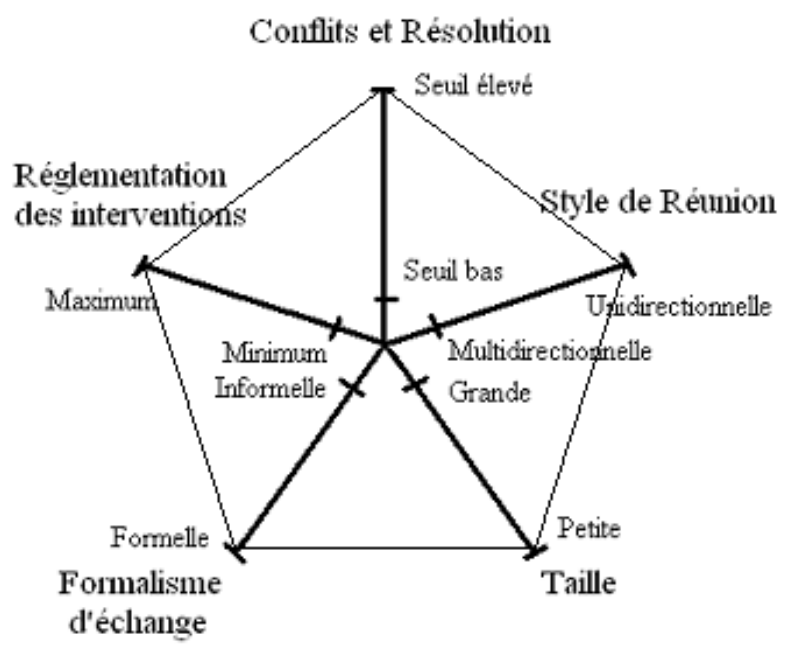

Figure 3 : Les cinq axes caractérisant le protocole d'interaction [12].

En appliquant le Denver Model, nous détaillons les quatre modes de coopération, en décrivant leur situation et leur protocole de l'interaction.

\subsection{Situation d'interaction}

Chaque polygone de la Figure 4 représente un mode de coopération : asynchrone, en session, en réunion et étroite. Les situations d’interaction explicitent ici les différences entre les modes. Toutes les situations ont en commun la taille du groupe et la localisation des membres (même ou des lieux différents). La différenciation se fait au niveau des trois axes "Coordination", "Temporalité" et "Dépendance"

o Coordination : les deux modes réunion (Figure 4 (3)) et étroite (Figure 4 (4)) correspond exactement à celles qui existent dans le cadre d'un projet (réunion et travail à la planche), c'est-à-dire planifiée. A l'opposé, un mode de coopération asynchrone (Figure 4 (1)) correspond au mode de travail autonome est spontané, c'est une réponse à une requête d'un autre membre ou est une offre spontanée à un membre. Entre les deux, le mode de coopération en session (Figure 4 (2)) est semiplanifiée : c-a-d planifiée de point de vue présence uniquement.

o Dépendance : la dépendance la plus forte est celle de la coopération étroite (Figure 4 (4)). En effet, dans ce mode les participants travaillent ensemble en temps réel 
sur des objets partagés. A l'opposé, dans les modes de coopération asynchrone (Figure 4 (1)) et en session (Figure 4 (2)) la dépendance est relâchée; tout le monde travaille de façon équivalente et indépendante. Pour le mode de coopération en réunion (Figure 4 (3)) la dépendance est possible.

o Temporalité : les trois modes de coopération en session, en réunion et étroite (Figure 4 (2), (3), (4)) exigent la connexion ou la présence des membres, c'est-àdire synchrone à l’ opposé du mode de coopération asynchrone (Figure 4 (1)).
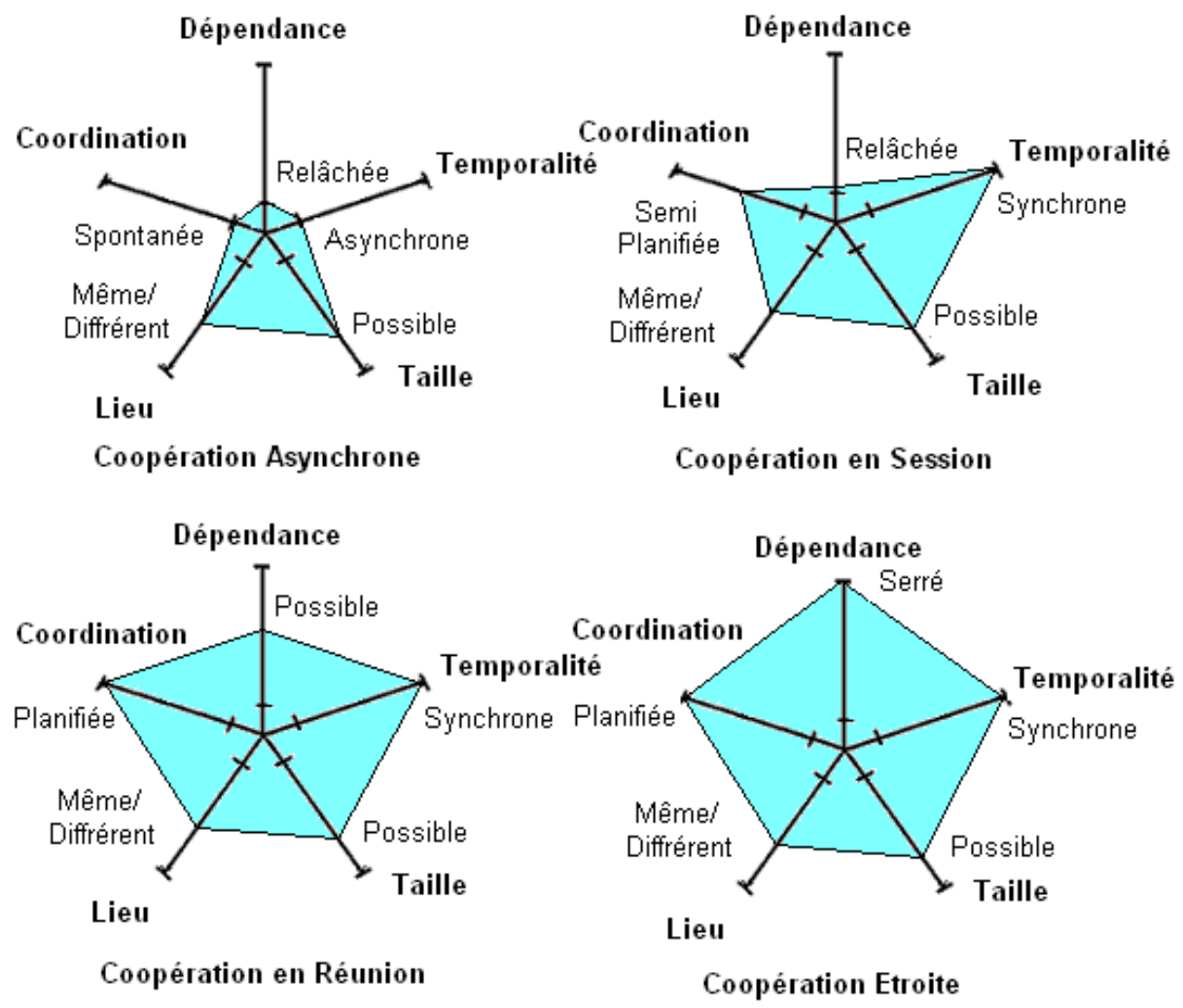

Figure 4 : Les situations d'interaction pour les quatre modes de coopérations 


\subsection{Protocole social de l'interaction}

En ce qui concerne les protocoles d'interaction, Chaque polygone de la Figure 5 représente un mode coopération : asynchrone, en session, en réunion et étroite. Tous ont en commun la caractéristique taille du groupe. La différenciation se fait au niveau des trois autres axes.

o Conflits et résolution : Les deux modes de coopération asynchrone (Figure 5 (1)) et en session (Figure 5 (2)) sont les modes de travail autonome, dans ces modes les membres sont libres pour résoudre d'éventuels conflits, ceci justifie alors la valeur basse attribuée à cette caractéristique. A l'opposé le mode coopération étroite (Figure 5 (4)), la valeur attribuée à cette caractéristique est élevé. Pour le mode de coopération en réunion (Figure 5 (3)) la valeur attribuée à cette caractéristique est possible, ceci ce justifie par le fait que ce mode correspond exacte aux réunions traditionnelles qui ne comporte pas des situations de concurrence ou d'opposition serrées.

o Style de réunion : la valeur attribuée à cette caractéristique dans les deux modes en réunion (Figure 5 (3)) et étroite (Figure 5 (4)) est unidirectionnelle, ceci ce justifie par le fait que ces modes sont contrôlés ou animés par le chef de projet et géré par un style de type tour de rôle. A l'opposé les autres modes asynchrone (Figure 5 (1)) et session (Figure 5 (4)) le style est multidirectionnel.

o Formalisme d'échange : Dans les deux modes asynchrone (Figure 4 (1)) et en session (Figure 4 (2)) les différents participants travail de façon autonome, ceci suppose l'existence de certaines formalité de communication entre eux. Dans les autres modes, la communication entre les participants est moins formelle par exemple un courrier électronique est plus formel qu'une note écrite sur un Post-It.

o Réglementation des interventions : Dans les deux modes de coopération asynchrone (Figure 5 (1)) et en session (Figure 5 (2)) la valeur attribuée à cette caractéristique est la plus basse pour exprimer le mode de travail autonome. A l'opposé, on trouve le mode de coopération étroite (Figure 5 (4)). Entre les deux, le mode de coopération en réunion. La valeur attribuée à cette caractéristique est possible, ceci exprime certaine liberté d'intervention tolérée aux participants. 


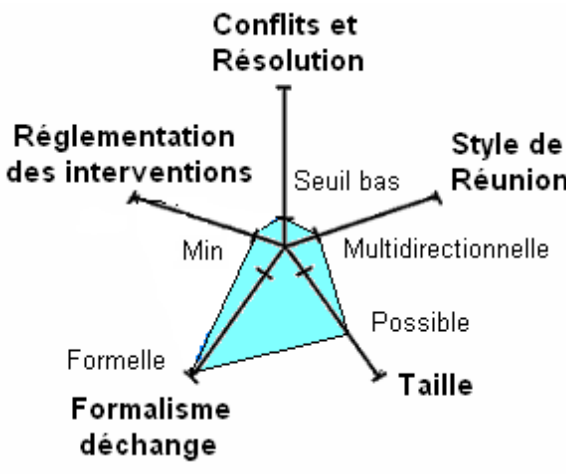

Coopération Asynchrone

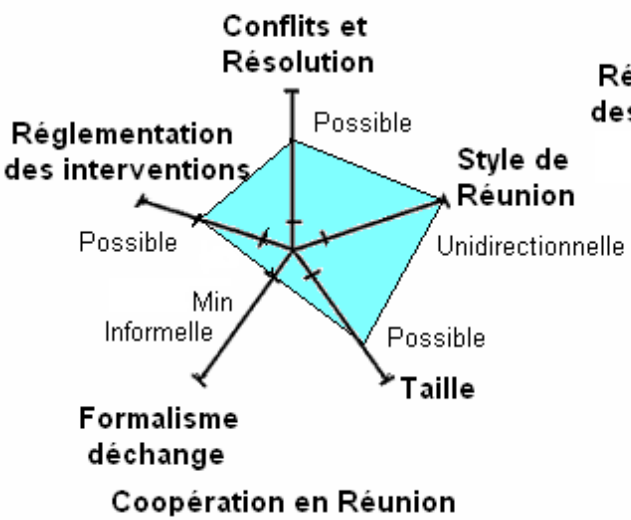

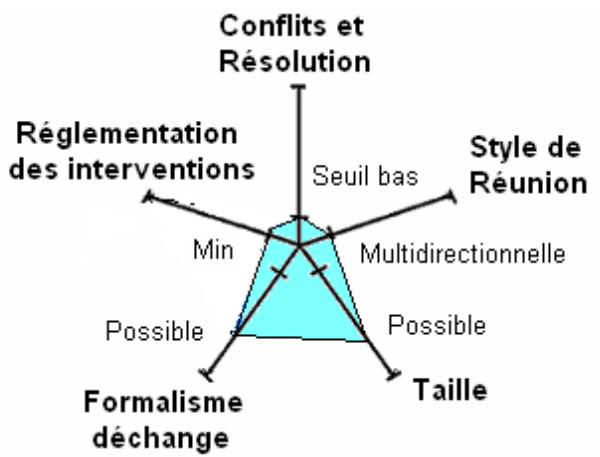

Coopération en Session

Conflits et

Résolution

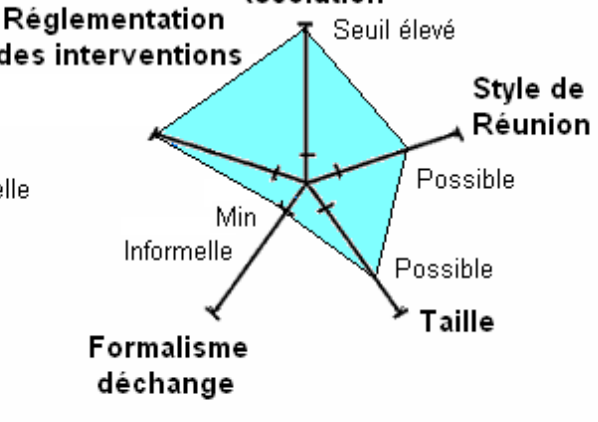

Coopération Etroite

Figure 5 : Les protocoles d'interaction des quatre modes de coopération

En appliquant le modèle Denver, nous avons modélisé les quatre modes de coopération, du point de vue des interactions, situations et protocoles. Dans la suite nous présentons notre expérimentation pour instancier et valider cette modélisation.

\section{APPLICATION : Expérimentation avec le système BSCW}

Conventionnellement la partie expérimentale est placée à la suite des parties consacrées à la modélisation et à la création de prototype (dans le but de valider ces dernières, d'en évaluer la pertinence et la performance, et de comparer une proposition nouvelle à des propositions antérieures qu'elle est censée améliorer). Dans notre cas, et 
comme vous pouvez l'imaginer, la réalisation d'un collecticiel fiable et valable en vue d'une expérimentation est impossible vu le temps imparti. Sans oublie que c'est un grand travail de groupe. En revanche, nous avons utilisé un collecticiel fiable prêt à l'emploi pour conduire une série d'expérimentation en vue d'instancier et valider nos modèles.

\subsection{Cadre de l'expérimentation}

L'expérimentation a pour cadre un projet de fin d'étude pour l'obtention d'un diplôme d'ingénieur en informatique industrielle à l'université du premier auteur. L'intitulé du thème était "Construction d'un modèle de simulation pour l'évaluation des performances d'un système de production : Cas de l'entreprise nationale des gaz industriels ENGI". Elle a été réalisée par deux étudiantes et diriger par leur directeur de stage, avec la collaboration de trois autres (un co-directeur de stage délégué par l'entreprise, le responsable de la production dans l'entreprise et un mathématicien). La durée du projet, et donc de l'expérimentation, était de six mois. La taille du groupe dans ce projet est de six personnes. En plus de ce projet, nous avons invité les étudiants en quatrième année de la même filière à expérimenter ce collecticiel dans le cadre de leurs travaux pratiques en modélisation et simulation. Le nombre total des participants dans cette expérimentation est 50.

\subsection{Objectifs de l'expérimentation}

Les expérimentations d’outils logiciels coopératifs dans le domaine de simulation sont rares; les seules expérimentations dont nous avons eu connaissance ont des objectifs différents des nôtres; les hypothèses qui les sous-tendent sont différentes de celles que nous essayons d'étudier : L'aspect coopératif d'un projet de simulation justifiant la prise en compte de la dimension de groupe dans les outils de simulation. L’objectif principal de notre expérimentation est :

o Instancier un collecticiel de simulation dans le but de valider les modèles élaborés.

o De mieux connaître les besoins d'une équipe de projet de simulation en termes d'outils de coopération.

\subsection{Choix de collecticiel : BSCW (Basic Support Cooperative Work)}

$\mathrm{Au}$ cours de cette phase de recherche sur le choix d'un collecticiel, nous avons comparé un ensemble de collecticiels qui nous semblaient adéquats pour 
l'implémentation d'un collecticiel simulation (BSCW, Buzzsaw, Groove, Team Wave et Sakai). Notre choix s'est porté sur le système BSCW pour les raisons suivantes :

o La simplicité et l'universalité de son accès dans un navigateur internet (web-based groupware). Le choix du Web comme plateforme permet de prendre en compte la diversité des machines, des systèmes d'exploitation, des différents types de réseaux. En effet, il dispense les utilisateurs de l'installation d'un logiciel client sur les machines dont ils désirent se servir. Elle leur permet également de travailler depuis des endroits différents.

o La gestion de projet partagé fondé sur des échanges asynchrones avec la possibilité de faire des échanges synchrones.

o Les fonctions de suivi de l'activité,

o La gratuité du serveur dans le cadre d'un usage éducatif,

o L'utilisation de ce serveur par de nombreux organismes dans un secteur similaire à notre cadre d'expérimentation [05].

Le BSCW [11], est un projet du GMD qui a pour objectif de définir des outils collaboratifs sur le Web qui fournissent des services collaboratifs inter-plates-formes à des groupes exploitant des technologies Web existantes. Ce système tente à fournir des caractéristiques basiques pour la coopération dans des services intégrés, accessibles depuis différentes plates-formes et qui ne demande pas à l'utilisateur d'adopter des applications spécifiques. Le BSCW Shared Workspace est un outil du BSCW, c'est une application coopérative centralisée intégrée à un serveur Web non modifié et accessible depuis des navigateurs Web standard. Ce système supporte la coopération à travers des 'espaces partagés' , formant des petits référentiels dans lesquels l'utilisateur peut télécharger des documents, suivre le fil d'une discussion, et obtenir des informations sur les activités précédentes des autres utilisateurs pour pouvoir coordonner son propre travail. Dans ce type de système c'est l'utilisateur qui doit prendre l'initiative de demander les informations nécessaires à la coordination de ses propres activités. Le système permet le partage de documents et de quelques formes de communication, mais il n’est pas en mesure d'accueillir des fonctionnalités collaboratives plus variées.

Nous reprenons une liste des fonctionnalités de BSCW [03] :

o Authentification. Les utilisateurs ont la possibilité de s’identifier auprès du serveur; cette identification est utile pour l'attribution de droits d'accès et l'historique des opérations. 
o Gestion des versions et verrouillage. Les fichiers déposés sur le serveur peuvent être versionnés (de façon linéaire ou arborescente) et verrouillés (seuls les propriétaires détient le droit de modifier le fichier).

o Forums de discussions. Ce sont des objets de BSCW qui contiennent des messages textuels; les messages sont organisés dans une structure arborescente où chaque message peut donner lieu à plusieurs réponses (situées à un niveau inférieur de la structure, le nombre de niveaux n'étant pas limité). Les forums de discussions sont des objets indépendants ou ils sont attachés à un fichier donné (annotation).

o Droits d'accès. Ils permettent de restreindre l'accès à certains fichiers ou certains répertoires pour des utilisateurs déterminés. Cette gestion des droits est dans BSCW très complexe d'utilisation (ex. héritage de droits, surcharge de droits, définition et modifications de catégories de droits) et donc potentiellement puissante.

o Fonctions de recherches. Elles sont destinés à retrouver des objets (ex. fichiers, personnes, réunions) de BSCW, principalement des fichiers, selon leur nom et/ou leurs attributs (ex. auteur, date de modification).

o Évaluation. Les utilisateurs peuvent évaluer chaque fichier (selon cinq options allant de "très mauvais » à " très bon »), le collecticiel se chargeant d'établir une moyenne de ces évaluations (ex. si un fichier a été jugé "très bon» par un utilisateur et «moyen » par un deuxième, la moyenne de ces deux évaluation est «bon »).

o Compression de fichiers. BSCW permet de compresser et de décompresser (au format zip) directement sur son serveur une liste de fichiers. Cela permet d'accélérer substantiellement les temps de dépôt et chargement de fichiers. Par exemple, l'utilisateur choisit de charger plusieurs fichiers depuis le serveur; il les compresse sur le serveur et charge ensuite le fichier compressé (appelé archive); une fois sur sa machine locale, il peut décompresser cette archive et récupérer les fichiers qui la constituent.

o Planification de réunion. Il est possible de planifier des réunions, et pour cela, de créer un objet spécifique (au même titre qu'un fichier) qui permet de déclarer des participants, un ordre du jour, un lieu et une date. Les participants sont en suite alertés par courrier électronique de la tenue de la réunion et peuvent répondre présent ou absent; la liste des personnes présentes ou absentes est mise à jour automatiquement et reste visible.

o Accès anonyme. L'accès anonyme permet à des utilisateurs occasionnels de visiter un projet et d'accéder (en lecture seulement) à des fichiers déposés par des utilisateurs enregistrés. 
o Personnalisation de l'interface. Le collecticiel s'affiche dans une fenêtre de navigateur. BSCW permet d'exploiter certaines fonctions de navigateur (ex. contrôle ActiveX d'Internet Explorer pour des "glisser-déposer ») pour en améliorer l'utilisation (ex. glisser-déposer). Il est également possible de définir des niveaux d'utilisation (ex. expert, débutant) qui agissent sur le nombre de fonctions proposés. Enfin, il est possible de changer la langue utilisée, c'est-à-dire non seulement les textes, mais également les icônes.

\subsection{Instauration des quatre modes de coopération}

La philosophie de fonctionnement du système BSCW et ces fonctionnalités nous ont permis d'instaurer parfaitement les deux modes de coopération asynchrone et session. L'instauration des deux autres modes de coopération est possible par l'utilisation des logiciels externes appropriés comme le NetMeeting. La figure 6 représente l'espace d'un participant qui discute la possibilité de l'utilisation du simulateur ARENA dans le projet en cours avec un autre membre via le logiciel NetMeeting.

L'adéquation du système BSCW dans les modes de coopération asynchrone et session a fait l'objet d'une expérimentation mené par Malcurat [10]. Le NetMeeting a aussi fait l'objet d'autres expérimentations qui montrent son adéquation avec le mode de coopération étroite [14] [15] [16]. Ces expérimentations nous permettent, d'une part, de confirmer le choix du collecticiel d'expérimentation, et d'autre part, d'argumenter l'approche de notre implémentation.

Dans la suite de ce document, nous présenterons un résumé sur notre approche d’implémentation du trèfle fonctionnel d'un collecticiel de simulation avec le système BSCW et MS-NetMeeting :

o Fonctionnalités de communication : En mode communication asynchrone, les membres peuvent communiquer directement par fils de discussion ; indirectement par les descriptions de fichiers et les commentaires de versions ou par courrier électronique. Le mode de communication synchrone se fait par adjonction logicielle (extension java).

o Fonctionnalités de coordination : Manuellement par fils de discussion. Deux possibilités : la première, semi-automatique, est l'envoi des dates de réunion par courrier électronique, réponse sur le serveur (présent ou absent). La deuxième, automatique cette fois-ci, par la possibilité de s'abonner aux rapports journaliers des changements intervenus.

o Fonctionnalités de production : les fonctionnalités de production sont assurées par l'utilisation des outils externes : Arena comme outil de simulation et MS-Office pour la production des documents (documents, schémas, présentations, etc.). Le 
partage d'application est non prévu dans le système BSCW; l'utilisation d'un outil externe, comme MS NetMeeting, peut combler ce manque dans une certaine mesure.

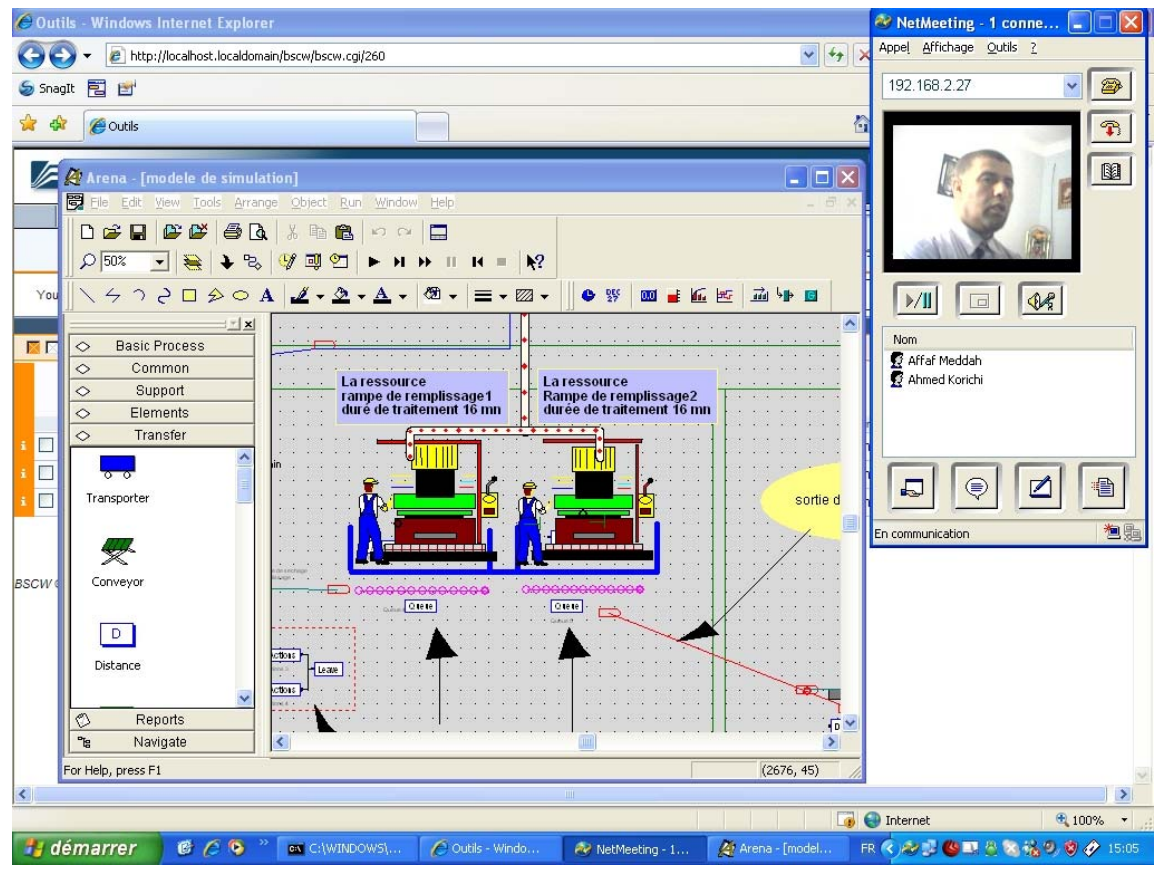

Figure 6 : BSCW \& NetMeeting en cours d'utilisation

\subsection{Limites de l'expérimentation}

Toute expérimentation, surtout dans un domaine où les composantes humaine et sociale sont si fortes, présente des limites dans la généralisation de ses conclusions. Citons au moins deux raisons : les données contextuelles de notre expérimentation sont très spécifiques (nombre des participants à l'expérimentation, relations entre les participants, nature du projet, etc.); elles illustrent une diversité combinatoire que seules plusieurs expérimentations pourraient approcher. D’autre part, la nature même de l'expérimentation motive les participants qui consentent des efforts sans doute inaccoutumés dans une pratique ordinaire. 
En plus de ces deux facteurs, notre expérimentation est sujette à des limitations qui lui sont propres et qui reflètent le décalage entre conditions expérimentales et conditions réelles. Il convient de les préciser :

o Le projet mené par les étudiants dans un contexte pédagogique bien différent du contexte réel. Les étudiants présentent fréquemment à leur encadreur l'avancement de leur travail. L’expérimentation du collecticiel ne pouvait interférer avec ce projet pédagogique; il n’était en particulier pas question de confronter les étudiants à l'utilisation d'un collecticiel et aux éventuelles difficultés techniques susceptibles de survenir.

o Les conditions privilégiées d'accès à Internet, en ce qui concerne la vitesse de transferts et la connexion permanente, favorise une utilisation dont la fréquence n’a sans doute pas d'équivalent dans le monde des professionnels d'aujourd'hui; les étudiants n’ont donc aucune peine à lire et à déposer fréquemment des fichiers volumineux. Là encore, pour minimiser ce décalage, nous pouvons considérer que les conditions de connexion sont appelées à évoluer pour le grand public, et rejoindre celles de l'expérimentation.

o La présence fréquente dans un même lieu et au même moment des étudiants n'était pas de nature à encourager la communication via le collecticiel. Il est plus naturel de s'adresser oralement à une personne que l'on a en face de soi plutôt que de recourir à une interface logicielle.

\subsection{Méthodes}

Tout projet coopératif requiert de la part des participants et dès son démarrage, un savoir méthodologique commun. Nous nous sommes employés à en fournir un, sous la forme d'un document que nous avons appelé guide de participant. Dans ce guide, nous avons été amenés à définir à la fois une méthode à destination des étudiantes, concepteurs du projet et une méthode à destination des expérimentateurs. Ce guide contient plusieurs points répondant à des exigences diverses :

o Structure organisationnelle : Identité et rôles des participants,

o Format des données utilisées,

o Structure des répertoires,

o Formes des requêtes dans chaque mode coopération,

o Répertoires publics. 


\subsection{Validation}

Bien que les fonctionnalités du BSCW et de NetMeeting nous permettent de supposer l'adéquation de cette implémentation pour la conduite d'un projet de simulation coopératif, il sera préférable de faire un sondage pour mesurer l'adéquation de cette implémentation. Deux approches ont été envisagées : La première est de demander aux participants de cette expérimentation de donner leurs opinions par le biais d'un questionnaire. La deuxième est de mettre en place une série de démonstrations interactives sur le Web et de demander aux visiteurs du site de donner leurs opinions par un questionnaire online. La seconde approche est écartée à cause du temps important nécessaire au développement.

Dans le questionnaire, et dans chaque mode de coopération, il a été demandé à chaque participant d'évaluer l'adéquation de l'implémentation en la notant sur une échelle de 1 à 5 . Nous demandons aux participants aussi d'évaluer leurs compétences en simulation et en technologie d'information. La sagesse rétrospective suggère que l'échelle 1 à 5 devrait être plus générale (Pour présenter une gamme plus large d'options) et que cette expertise devrait avoir d'autres facteurs plutôt qu'un simple nombre.

Le Tableau 1 représente les résultats chiffrés de ce sondage pour chaque mode de coopération (nombre de répondants par note), le tableau 2 représente le pourcentage des cinq notes pour chaque mode de coopération et le pourcentage relatif à l'implémentation complète. La Figure 7 et 8 sont les représentations graphiques des résultats du deuxième tableau.

Bien que le nombre des participants dans cette expérimentation soit plus ou mois petit, le résultat de cette expérience est parfaitement clair

\begin{tabular}{|c|c|c|c|c|c|c|}
\hline Mode de coopération & & re d & ponc & s pa & & Total \\
\hline Note $/ 5 \rightarrow$ & 1 & 2 & 3 & 4 & 5 & 50 \\
\hline Asynchrone & 00 & 00 & 00 & 10 & 40 & 50 \\
\hline Session & 00 & 00 & 04 & 41 & 05 & 50 \\
\hline Réunion & 00 & 00 & 06 & 24 & 20 & 50 \\
\hline Etroite & 00 & 00 & 37 & 09 & 04 & 50 \\
\hline Total cumulé $\rightarrow$ & 00 & 00 & 47 & 84 & 69 & 200 \\
\hline
\end{tabular}

Tableau 1 : Nombre de répondants par note 


\begin{tabular}{|c|c|c|c|c|c|}
\hline \multirow{2}{*}{$\begin{array}{l}\text { Mode de coopération } \\
\qquad \text { Note } / 5 \rightarrow\end{array}$} & \multicolumn{5}{|c|}{ Pourcentage des répondants par notes } \\
\hline & 1 & 2 & 3 & 4 & 5 \\
\hline Asynchrone & $0 \%$ & $0 \%$ & $0 \%$ & $20 \%$ & $80 \%$ \\
\hline Session & $0 \%$ & $0 \%$ & $8 \%$ & $82 \%$ & $10 \%$ \\
\hline Réunion & $0 \%$ & $0 \%$ & $12 \%$ & $40 \%$ & $48 \%$ \\
\hline Etroite & $0 \%$ & $0 \%$ & $74 \%$ & $18 \%$ & $8 \%$ \\
\hline Evaluation Cumulé $\rightarrow$ & $0 \%$ & $0 \%$ & $23 \%$ & $42 \%$ & $35 \%$ \\
\hline
\end{tabular}

Tableau 2 : Pourcentage des répondants par note

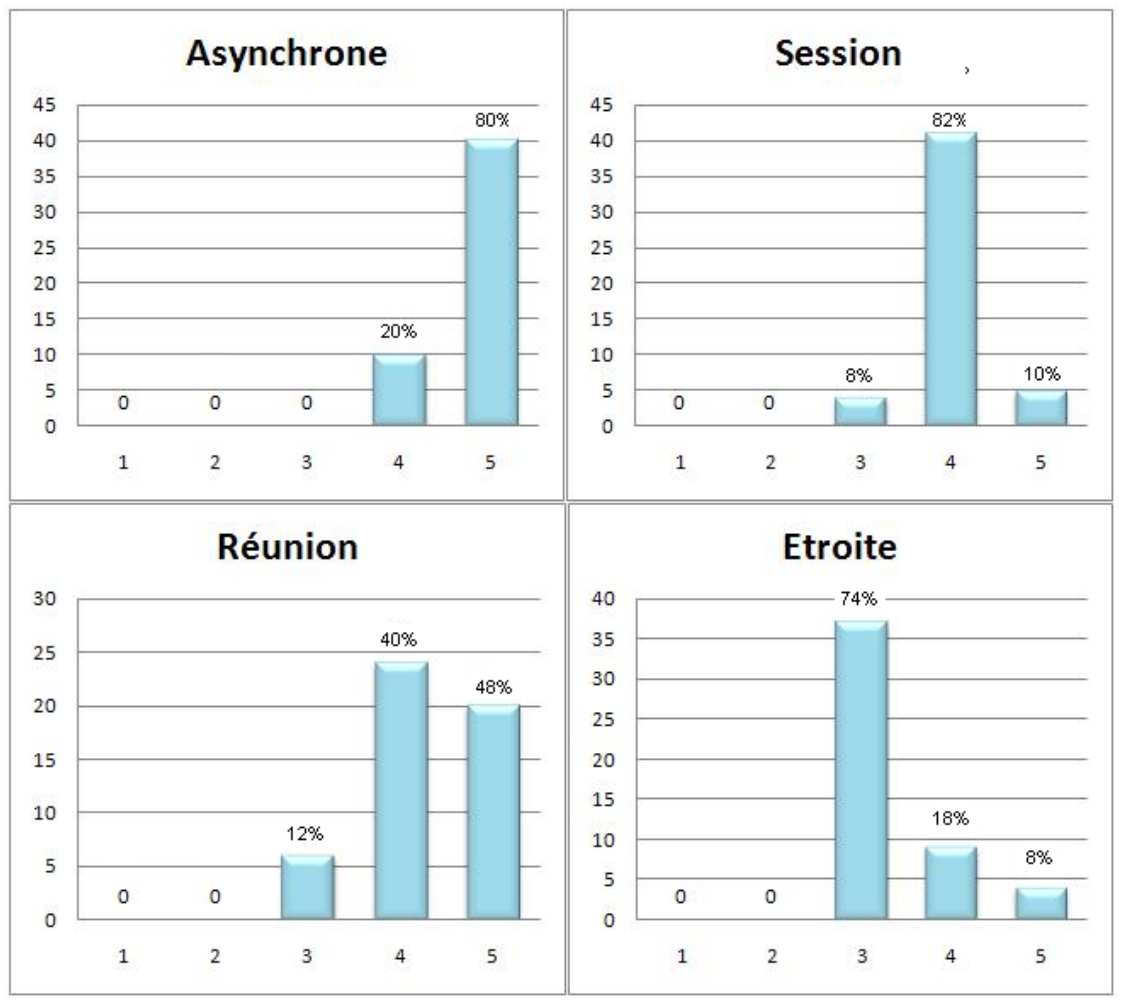

Figure 7 : Evaluation des quatre modes de coopération 


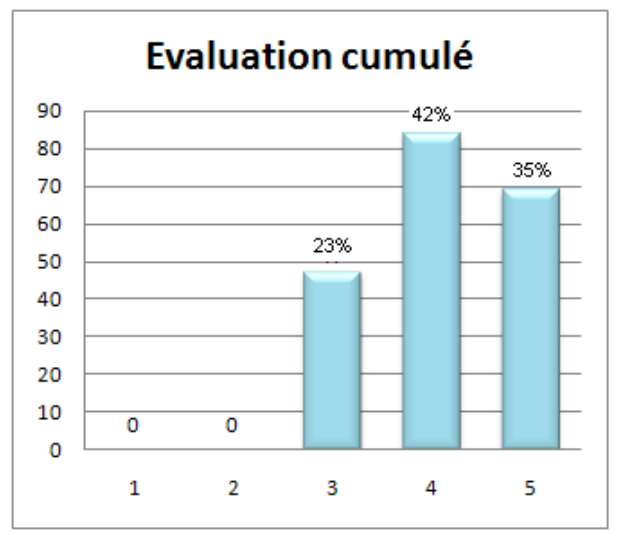

Figure 8 : Evaluation cumulée de l'implémentation

\section{Conclusion}

Dans ce papier, nous avons proposé une modélisation des interactions de coopération dans un projet de simulation qui comprend quatre modes de coopération. Ces derniers ont été définis à partir de la méthode de conduite d'un projet de simulation. Nous les avons ensuite caractérisés à l'aide du Denver Model, puis programmées au sein du système BSCW. L'approche que nous avons employée pour la création des modèles que nous avons présentés semble être suffisamment flexible pour permettre des évolutions et des développements futurs. En effet les modèles que nous avons proposés peuvent être largement exploitées dans un autre créneau, comme celui de l'enseignement coopératif de la simulation.

\section{Bibliographie}

[01] Belattar, B., Korichi A. June 25-29 2001. GOMSE: A Group Oriented Modeling and Simulation Environment. Proceedings of the ACS/IEEE International Conference on Computer Systems and Applications. Beirut Lebanon. 4 pages.

[02] Belattar, B., Korichi A. (2001). Intégration des Concepts du Travail Coopératif Assisté par Ordinateur en Simulation. Conférence Internationale JIM’2001, Metz France, 4-7 Juillet.

[03] BSCW, 2007. Site WEB du système BSCW (URL : http://bscw.gmd.de), Visité en Jnavier 2007. 
[04] Banks, J., ed., 1998. Handbook of Simulation: Principles, Methodology, Advances, Applications, and Practice, John Wiley, New York.

[05] González V.R., García de la Santa1 A., Gorghiu G., Gorghiu L.M. 2005. "BSCW as a support system for distance teacher training, in: Recent Research Developments in Learning Technologies, Proceedings of the Third International Conference on Multimedia \& ICT's in Education, vol. 2, pp. 696-701.

[06] John A. Miller, Gregory Baramidze, Paul A. Fishwick and Amit P. Sheth. 2004. Investigating Ontologies for Simulation Modeling. Proceedings of the 37th Annual Simulation Symposium (ANSS'04), Arlington, Virginia April. pp. 55-71.

[07] Juillien , B., Grimaud F., 1997. Simulation des systèmes de production avec ARENA. Support de Cours. Ecole des Mines Saint Etienne, France.

[08] KLC (Dictionnaire de la maîtrise d'ouvrage des projets informatiques) (2007). , Site Web accessible à l'url: http://www.klc.fr/FR/METHO/DicoSI_IE_FR.html. Visité en Janvier 2007.

[09] Laurillau, Y., 2002. Conception et réalisation logicielles pour les collecticiels centrées sur l'activité de groupe : le modèle et la plate-forme Clover. Thèse de doctorat de l'université Joseph Fourier - Grenoble I, Laboratoire de Communication Langagière et Interaction Personne - Système Fédération IMAG, 10 Septembre. 216 pages.

[10] Malcurat, O., 2001. Spécification d'un environnement logiciel d'assistance au travail coopératif dans le secteur de l'architecture et du BTP. Thèse de doctorat, Sciences pour l'architecture. Institut National Polytechnique de Lorraine. 153 pages.

[11] Payet, D., 2003. L'enrichissement de message comme support pour la composition logicielle. Thèse de doctorat de l'université de Montpellier II, 04 Décembre. 228 pages.

[12] Salvador, T., Scholtz, J., Larson, J., 18, January 1996. The Denver Model for Groupware Design. SIGCHI Bulletin Vol 28 No 1. 52-58.

[13] Tarpin-Bernard, F., July 1997. Travail Coopératif Synchrone Assisté par Ordinateur Approche AMF-C. Thèse de Doctorat. University of Lyon.

[14] Taylor, S.J.E., 2000. NetMeeting: a tool for collaborative simulation modelling I. J. of Simulation Systems, Science \& Technology Vol. 1 No 1-2:59-68.

[15] Taylor, S.J.E., Robinson, S. and Ladbrook, J., 2005. An Investigation into the Use of NetConferencing Groupware in Simulation Modelling. Journal of Computing and Information Technology, 13, 1, pp. 1-10.

[16] Taylor, S.J.E., Robinson, S. and Ladbrook, J., 2003. Towards Collaborative Simulation Modelling: Improving Human-to-Human Interaction Through Groupware. Proceedings of the 17th European Simulation Multiconference (ESM 2003). SCS Europe Bvba, Erlangen, Germany. pp. 474-482. 
[17] Wladimir, A. F., Hirata C. M., Edgar T. Y. 2004. GroupSim: A Collaborative Environment for Discrete Event Simulation Software Development for the World Wide Web. SagePub, Vol. 80, Num 6. pp 257-272.

\section{Biographie}

Dr. Ahmed Korichi est maitre de conférences en informatique au département de mathématique et d'informatique de l'université d'Ouargla en Algérie. Ses thèmes de recherches sont le TCAO, simulation et modélisation. II dirige des mémoires d'ingénieurs en Informatique à l'université de Ouargla.

Dr. Brahim Belattar est maître de conférences en informatique au département d'informatique de l'université de Batna. Ses thèmes de recherche sont l'enseignement à distance, le travail coopératif et l'usage de l'Internet, simulation et modélisation. II dirige des thèses de doctorat et de magister en Informatique à l'université de Batna. 